Supporting Information

\title{
Enhanced superconductivity induced by the hexagonal-array-cooling-shrinkage effect
}

Zhigang LAt, „, *, Tianle Wang ${ }^{\dagger}$, Shangshen Fengs, *, Minghu Fangl/, *, Shijie Shen ${ }^{\dagger}$, Zhaorong Yang ${ }^{\perp}$, and Bingqing We ${ }^{\star}$, *

†School of Pharmaceutical \& Materials Engineering, Taizhou University, Taizhou 318000,

China

¥Department of Mechanical Engineering, University of Delaware, Newark, DE 19716, USA

\$School of Science, Zhejiang Agricultural and Forestry University, Hangzhou 311300, China

"Physics Department, Zhejiang University, Hangzhou 310027, China

${ }^{\perp}$ High magnetic field laboratory, Chinese Academy of Sciences, Hefei 230031, China

* Corresponding authors:

E-mail address: lizhigang@issp.ac.cn (Z.L.); fengss@zafu.edu.cn (S. F.);

mhfang@zju.edu.cn (M. F.); weib@udel.edu (B.W.) 


\section{Silicon Nb Film Au wire PS}

a

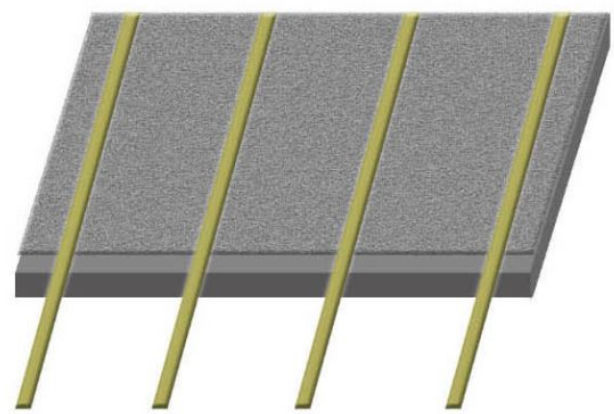

b

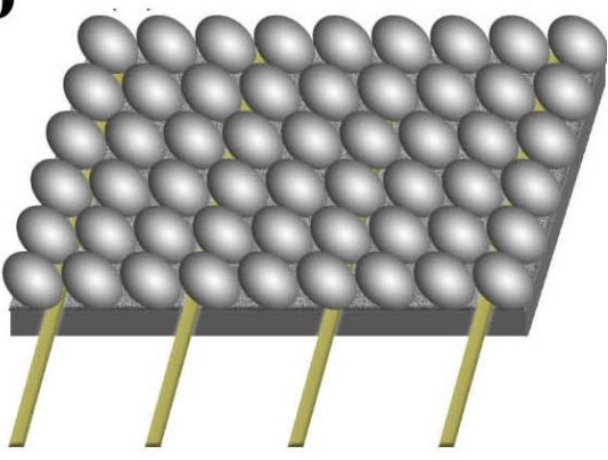

C
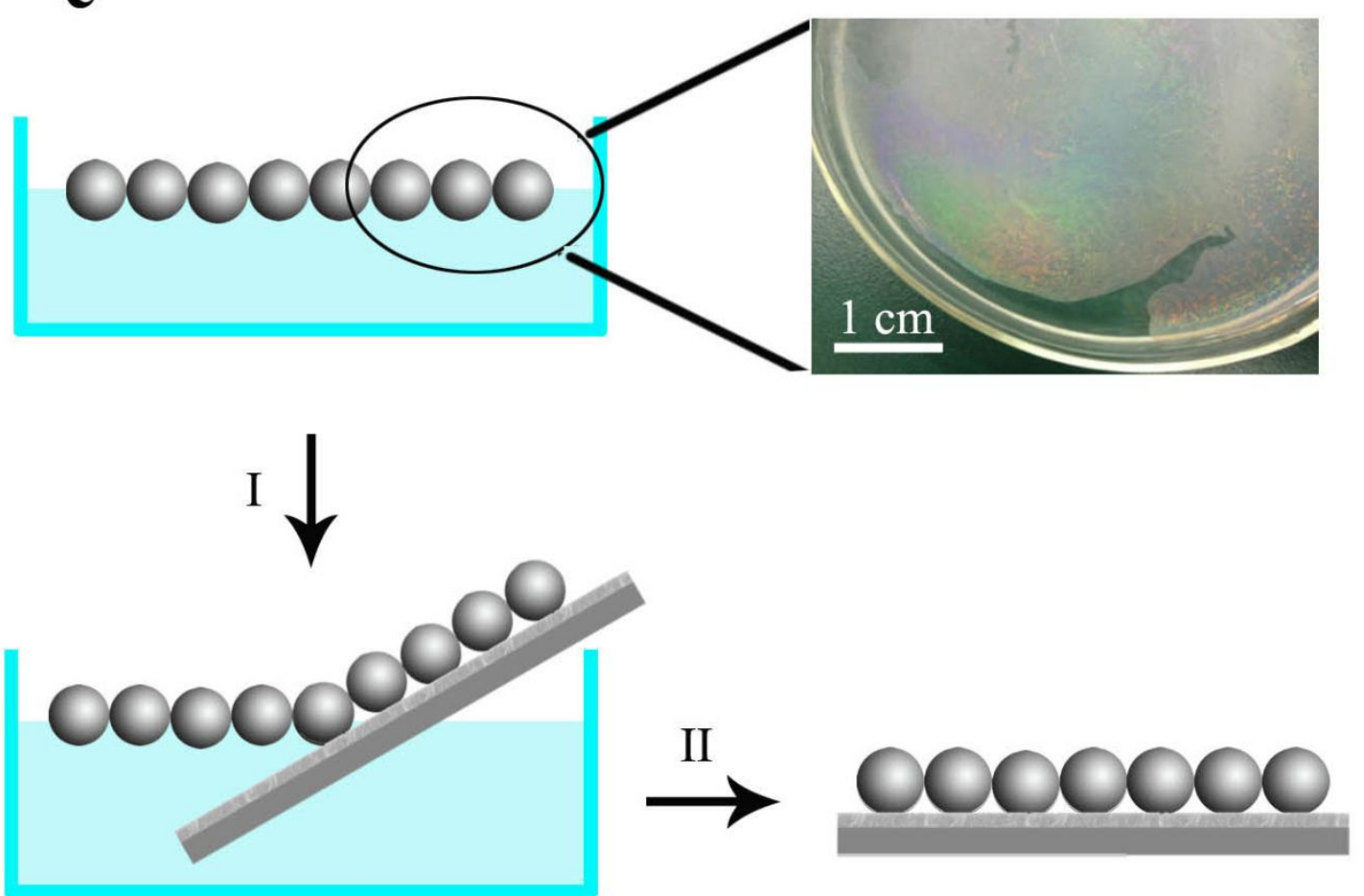

Figure S1. Illustration of the $\mathrm{Nb}$ film (a) and $\mathrm{PSs} / \mathrm{Nb}$ hybrid (b) with Au electrodes. (c) Combination process of PSs on the $\mathrm{Nb}$ film. A PS colloidal array is first prepared via a self-assembly process in a petri dish. Then, the PS array is transferred onto a pre-fabricated $\mathrm{Nb}$ film substrate (I). Subsequently, the excess water on the surface of the PS layer was absorbed by a filter paper, the PSs and $\mathrm{Nb}$ film are bonded together under atmospheric pressure. 

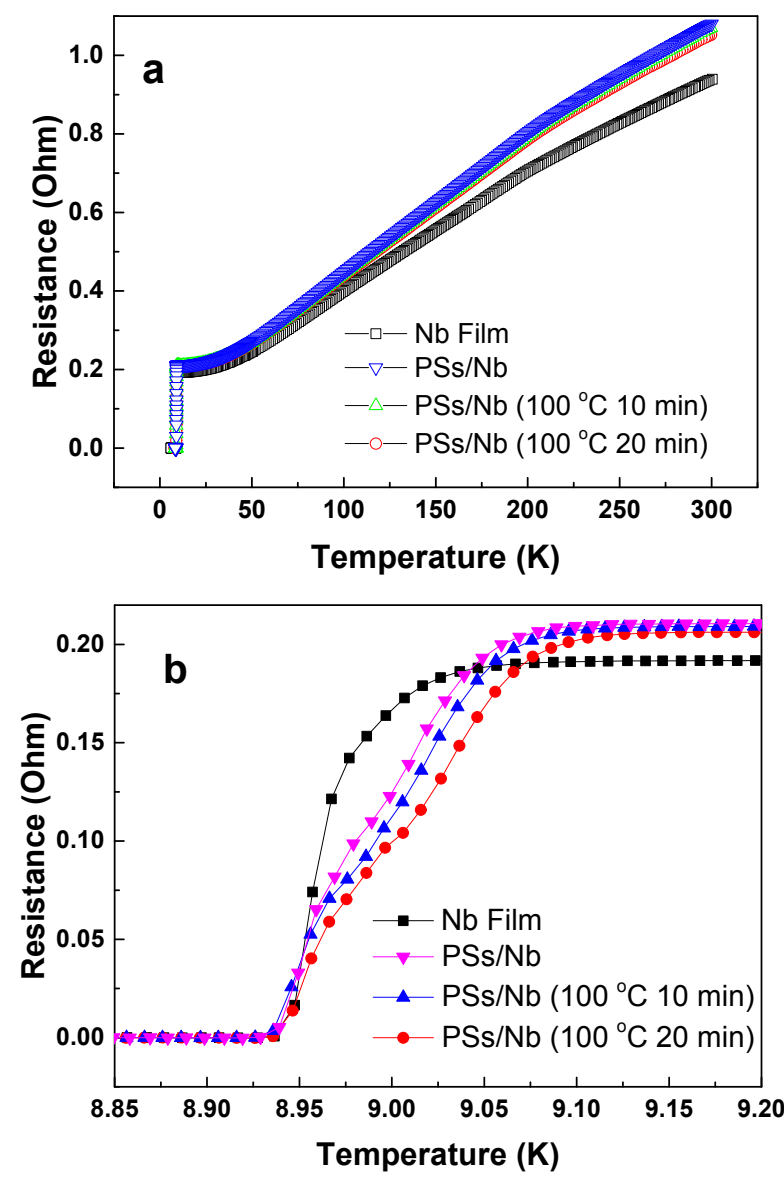

Figure S2. The raw data of Fig. 2a without normalization. (a) 300-8.2 K; (b) 9.2-8.85 K.

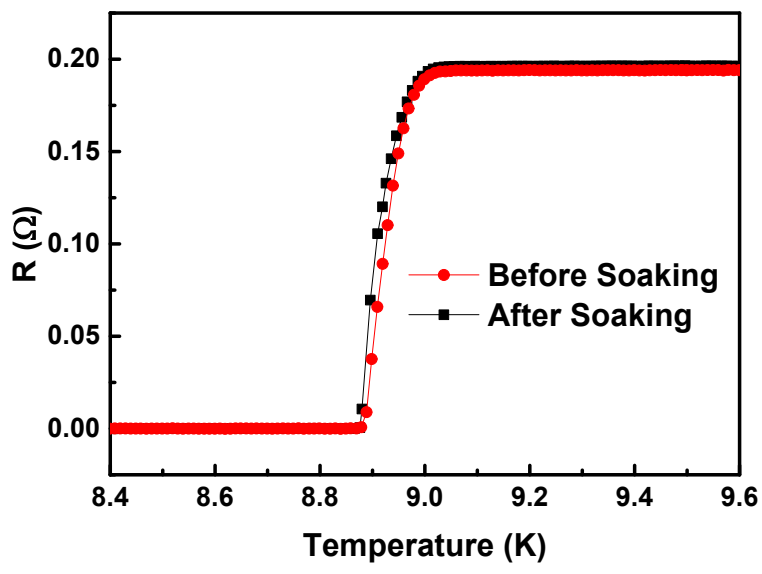

Figure S3. The R-T curves of parallel $\mathrm{Nb}$ film before and after soaking. 

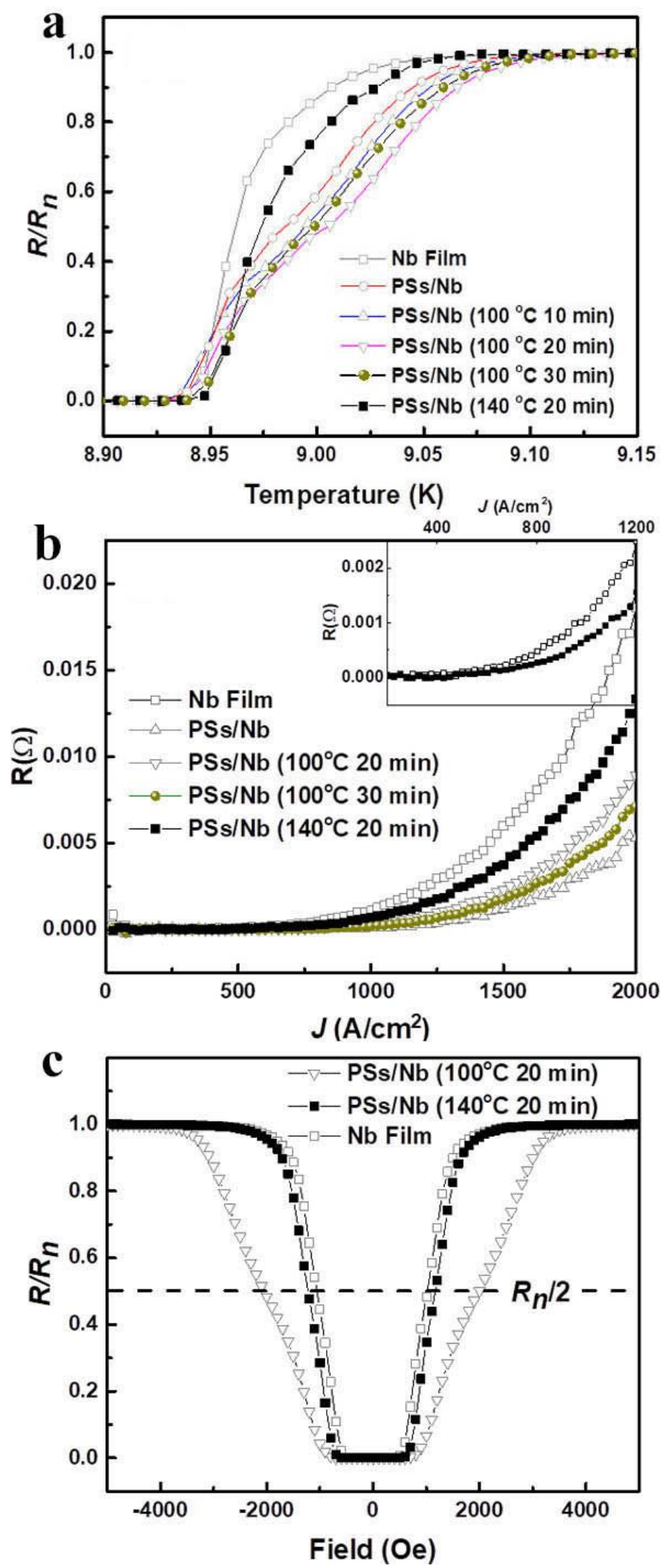

Figure S4. The enhanced superconductivity of the hybrid with different heat treatments. (a) R-T curves, (b) I-V curves at $8.9 \mathrm{~K}$, the inset is the amplification of $\mathrm{Nb}$ film and $\mathrm{PSs} / \mathrm{Nb}\left(140{ }^{\circ} \mathrm{C} 20 \mathrm{~min}\right)$. (c) $\mathrm{R}-\mathrm{H}$ curves at $8.9 \mathrm{~K}$. 


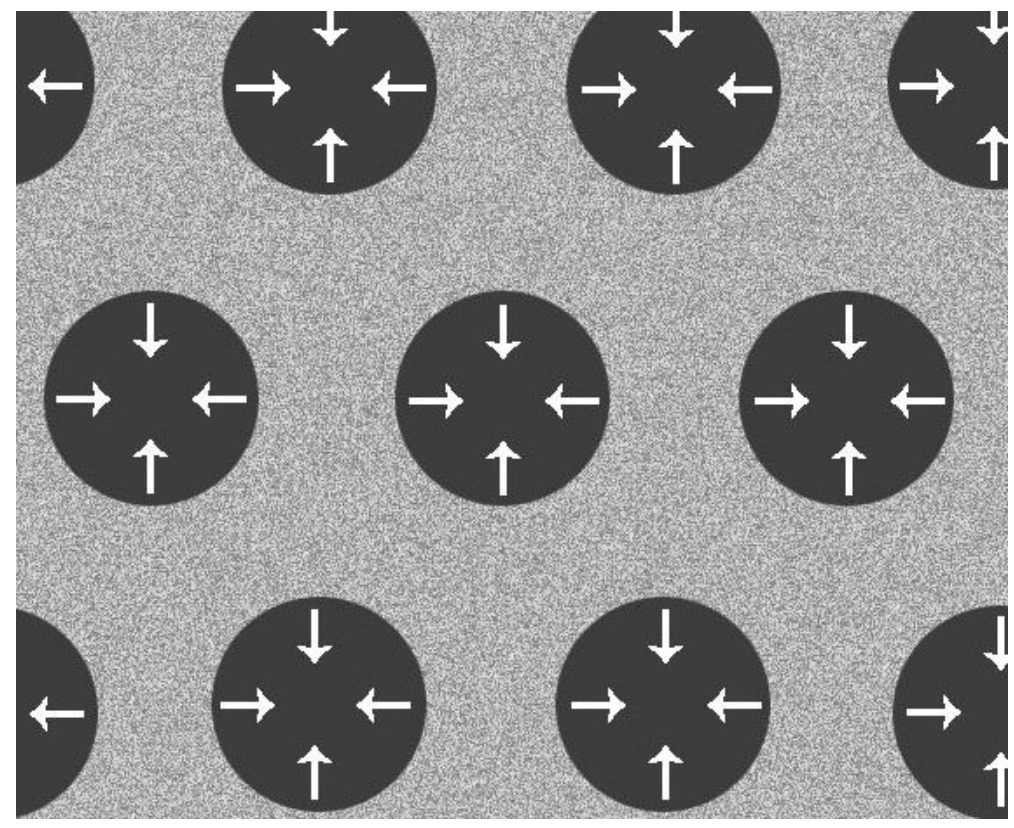

Figure S5. A schematic force diagram of the PSs/Nb hybrid. The black circles are the contact areas between PSs and the $\mathrm{Nb}$ film, and the white arrows indicate the direction of compression stress.

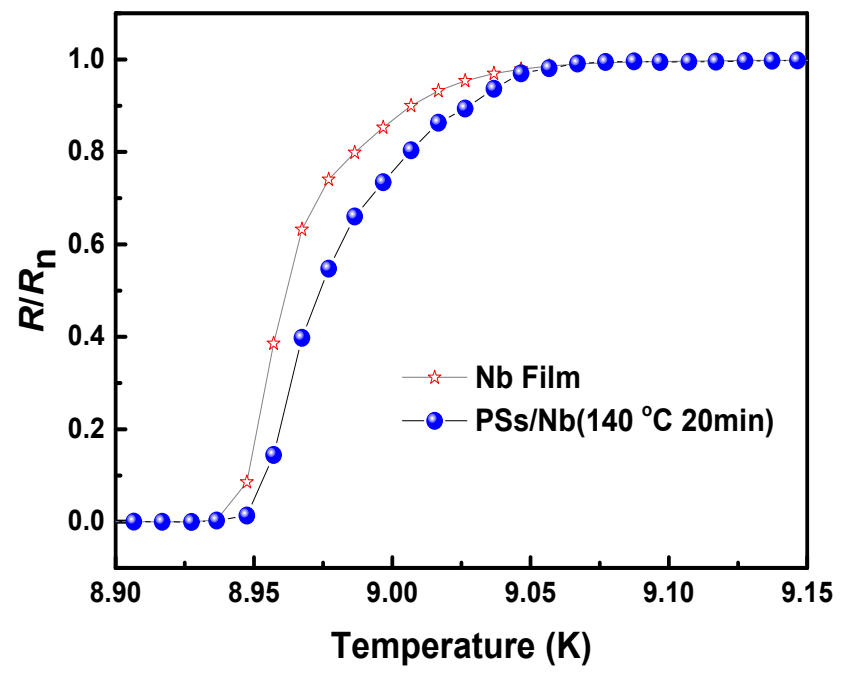

Figure S6. $\mathrm{R}-\mathrm{T}$ curves of the $\mathrm{Nb}$ film and $\mathrm{PSs} / \mathrm{Nb}\left(140{ }^{\circ} \mathrm{C} 20 \mathrm{~min}\right)$ hybrid. 

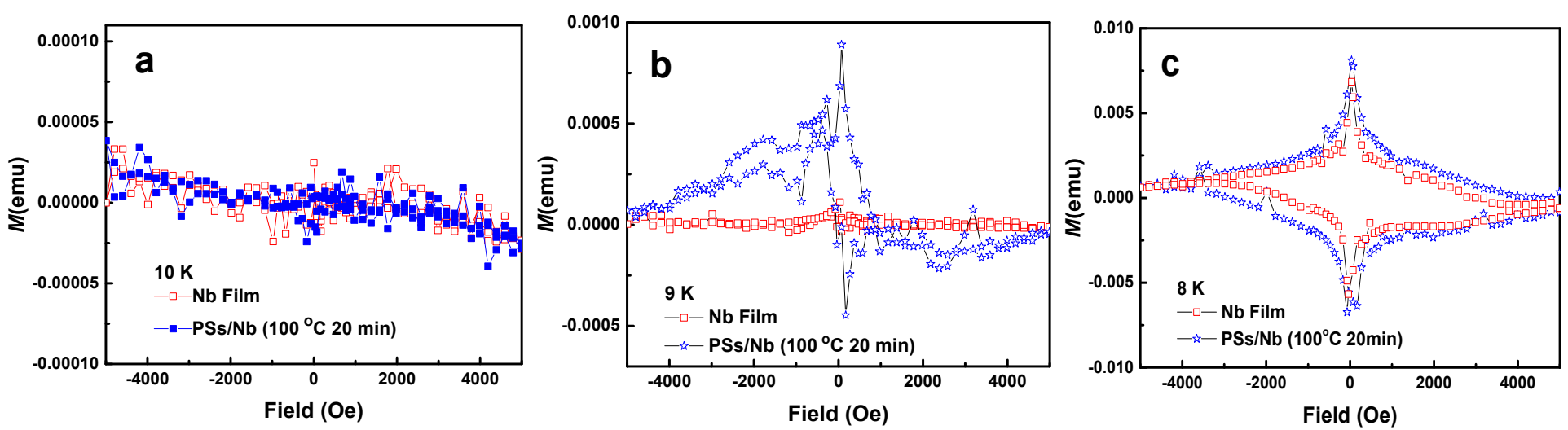

Figure S7. The hysteresis loops of the $\mathrm{Nb}$ film and $\mathrm{PSs} / \mathrm{Nb}\left(100^{\circ} \mathrm{C} 20 \mathrm{~min}\right)$ at different temperatures (a) $10 \mathrm{~K}$, (b) $9 \mathrm{~K}$, and (c) $8 \mathrm{~K}$.
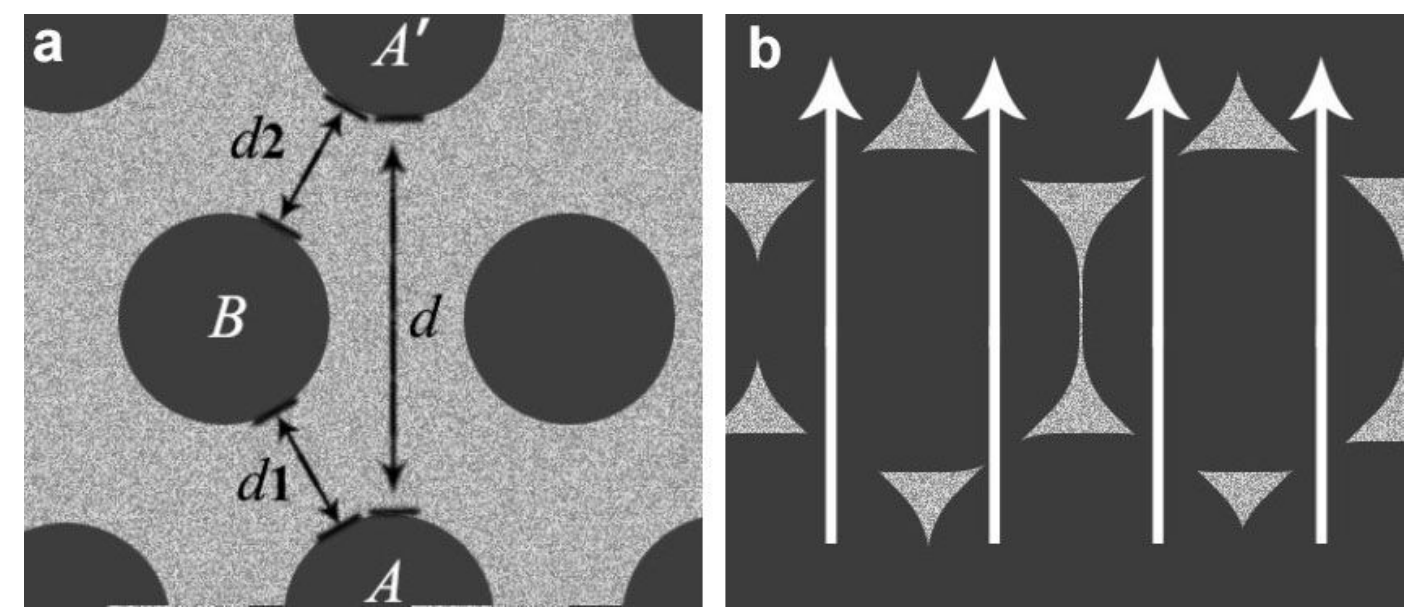

Figure S8. A schematic for contact area size of the PSs/Nb hybrid. (a) $d 1+d 2>d$; (b) the large contact areas. The white arrows indicate the direction in which the electrons are moving. 

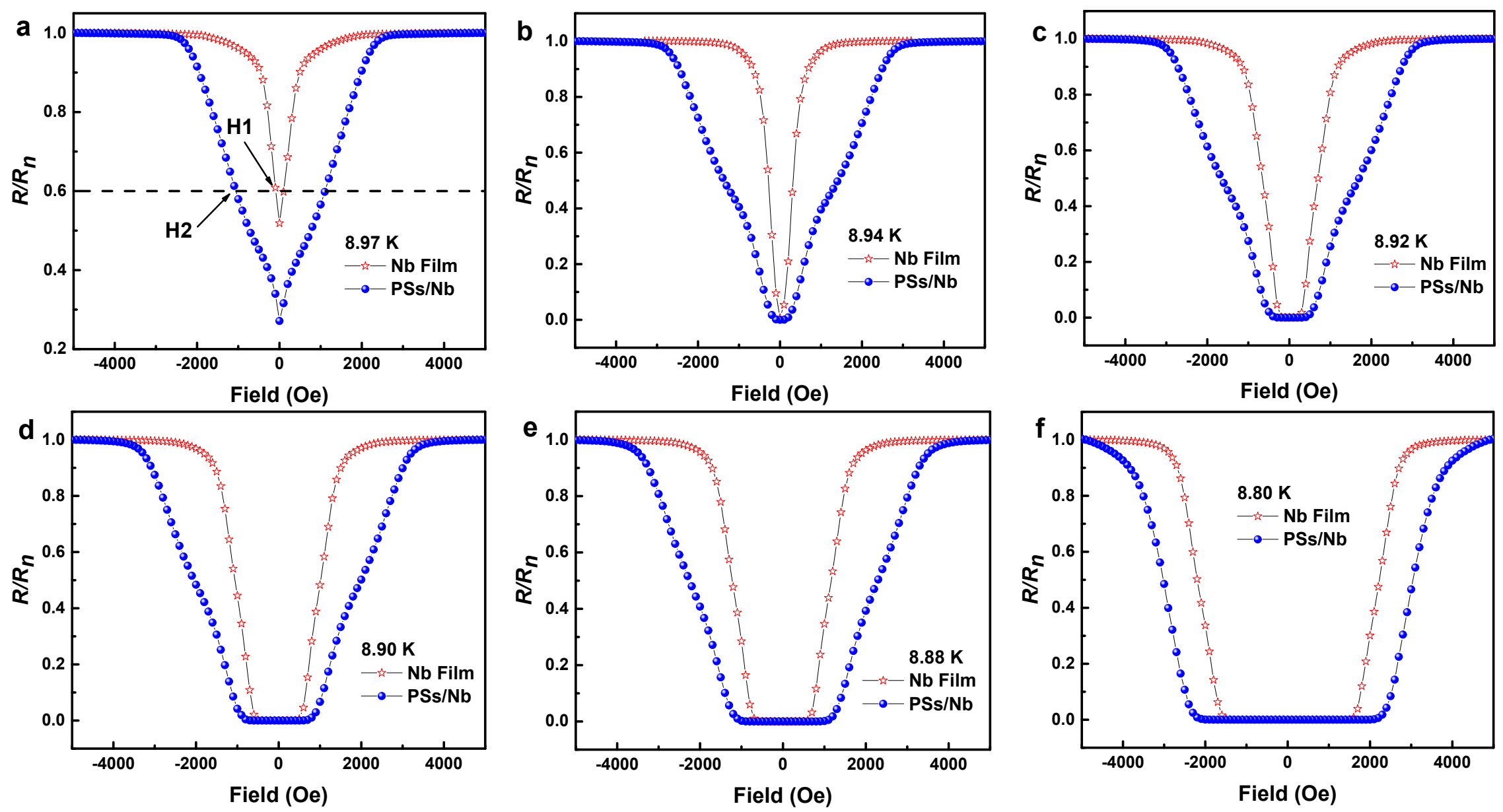

Figure S9. The R-H curves of the $\mathrm{Nb}$ film and PSs/Nb hybrid at different temperatures. (a) $8.97 \mathrm{~K}$ (b) $8.94 \mathrm{~K}$, (c) $8.92 \mathrm{~K}$, (d) $8.90 \mathrm{~K}$, (e) $8.88 \mathrm{~K}$, and (f) $8.80 \mathrm{~K}$. 

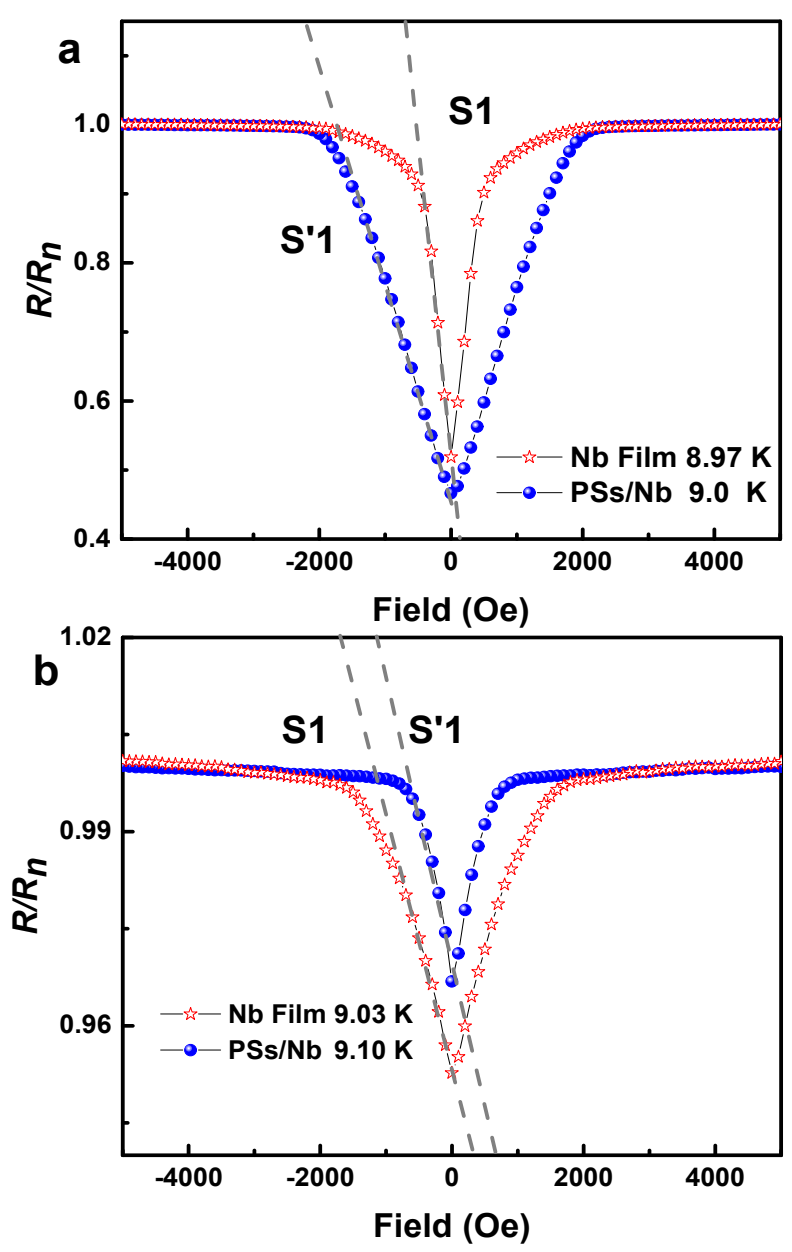

Figure S10. The R-H curves of the $\mathrm{Nb}$ film and $\mathrm{PSs} / \mathrm{Nb}$ hybrid with similar resistance. (a) $\mathrm{Nb}$ film $(8.97 \mathrm{~K})$ and PSs/Nb hybrid $(9.0 \mathrm{~K})$. (b) $\mathrm{Nb}$ film $(9.03 \mathrm{~K})$ and PSs/Nb hybrid $(9.10 \mathrm{~K})$. Here, S1 and $\mathrm{S}^{\prime} 1$ correspond to the slope straight lines of the $\mathrm{Nb}$ film and $\mathrm{PSs} / \mathrm{Nb}$ hybrid, respectively. 